$56^{\text {ème }}$ Congrès de la SFMBCB, 03014 (2011)

DOI: $10.1051 / \mathrm{sfmbcb} / 20115603014$

(c) Owned by the authors, published by EDP Sciences, 2011

\title{
Méthodes d'imagerie in vivo sur le petit animal : intérêt en ingénierie tissulaire osseuse
}

\author{
Catros $S^{1,2}$, Ziane $S^{2}$, Lalande $C^{2}$, Arnault $I^{2}$, Rousseau $B^{4}$, Miraux $S^{3}$, Chassande $O^{2}$, \\ Guillemot $\mathbf{F}^{2}$, Fricain $\mathbf{J C}^{1,2}$ \\ ${ }^{1}$ Faculté de Chirurgie dentaire, Université Victor Segalen, Bordeaux, France \\ ${ }^{2}$ Inserm U577 Biomatériaux et Réparation tissulaire, Université Victor Segalen, Bordeaux, \\ France \\ ${ }^{3}$ Centre de Résonance magnétique des Systèmes biologiques, Université Victor Segalen, \\ Bordeaux, France \\ ${ }^{4}$ Animalerie A2, Université Victor Segalen, Bordeaux, France \\ sylvaincatros@hotmail.com
}

Parmi les différentes étapes d'élaboration et de validation d'un produit d'ingénierie tissulaire pour la reconstruction osseuse, l'expérimentation in vivo est encore actuellement un temps nécessaire qui permet une évaluation dans des conditions proches de la physiologie (Muschler 2009). Ces études ont longtemps été réalisées uniquement à travers des analyses histologiques post mortem, ce qui nécessitait des cohortes importantes afin d'étudier les différents temps de cicatrisation. L'introduction de méthodes d'imagerie in vivo non invasives dédiées permet actuellement d'étudier plus finement les différentes étapes de l'intégration du matériau et de la cicatrisation chez le petit animal (souris, rat), en utilisant un seul groupe étudié à plusieurs temps, sous anesthésie générale (isofluorane). L’objectif de cette note technique est d'exposer les progrès technologiques récents dans le domaine de l'imagerie in vivo pour la recherche, à travers trois méthodes utilisées dans le domaine de l'ingénierie tissulaire osseuse : le microscanner, la micro-IRM et la bio-luminescence.

La principale application du micro-scanner $(\mu \mathrm{CT})$ in vivo est l'étude de l'os et des tissus minéralisés (Schambach 2009), même si des applications récentes en imagerie cardiopulmonaire et tumorale ont été décrites (Badea 2008). Concernant les applications osseuses, le $\mu$-CT in vivo chez la souris ( $\mu$ CT General Electric ${ }^{\circ}$ ) permet l'appréciation de la morphologie et la quantification de la densité osseuse, de façon longitudinale, sans sacrifice de l'animal. La limite de résolution in vivo de $50 \mu \mathrm{m}$ est liée aux impératifs de dosimétrie puisque les $\mu \mathrm{CT}$ ex-vivo permettent d'atteindre des résolutions de $8 \mu \mathrm{m}$. Il a préalablement été démontré que les quantifications morphologiques obtenues sont significativement corrélées avec l’histomorphométrie conventionnelle sur coupes décalcifiées (Malivaud 2008).

La micro-imagerie par résonnance magnétique ( $\mu$-IRM, Bruker ${ }^{\circledR}$, Germany) possède une résolution de 100 à $200 \mu \mathrm{m}$. Elle peut être utilisée pour surveiller l'évolution de la réaction inflammatoire postopératoire ou bien la prolifération tumorale au sein des tissus mous. Par ailleurs, l'utilisation de cellules porteuses de particules ferromagnétiques (Versatile Ultra Small Paramagnetic Iron Oxide : VUSPIO) permet d'apporter du contraste aux images et de localiser précisément les cellules dans les matériaux implantés (Bouzier-Sore 2009).

L'utilisation de cellules luminescentes in vivo dans la construction de matériaux composites cellularisés permet un suivi longitudinal de la prolifération grâce à des acquisitions faites sur un photon imageur (De Boer 2006, Degano 2007). Les cellules utilisées sont transfectées par une enzyme (la luciférase) qui, en présence de son substrat (la luciférine) permet à la cellule hôte de produire des photons. Après anesthésie, le substrat est injecté par voie intrapéritonéale et vient réagir avec l'enzyme, ce qui permet de localiser et de quantifier les cellules implantées par l'intermédiaire d'une caméra à haute résolution (Photon Imageur Biospace $^{\odot}$, France). La quantité de photons émis est en rapport direct avec la prolifération cellulaire (Hara 2008).

Ces méthodes peuvent être couplées et comparées entre elles ainsi qu’avec les résultats de l’analyse histologique post-mortem. 